\title{
Histone Methylations Define Neural Stem/Progenitor Cell Subtypes in the Mouse Subventricular Zone
}

\author{
Zhichao Zhang ${ }^{1,2} \cdot$ Adeel Manaf $^{2} \cdot$ Yanjiao $^{2 i^{2,3}} \cdot$ Sonia Peña Perez ${ }^{2} \cdot$ Rajikala Suganthan $^{2} \cdot$ John Arne Dahl $^{2}$. \\ Magnar Bjørås ${ }^{2,4} \cdot$ Arne Klungland $^{2,3}$ (1)
}

Received: 27 May 2019 / Accepted: 3 September 2019 / Published online: 25 October 2019

(C) The Author(s) 2019

\begin{abstract}
Neural stem/progenitor cells (NSPCs) persist in the mammalian brain throughout life and can be activated in response to the physiological and pathophysiological stimuli. Epigenetic reprogramming of NPSC represents a novel strategy for enhancing the intrinsic potential of the brain to regenerate after brain injury. Therefore, defining the epigenetic features of NSPCs is important for developing epigenetic therapies for targeted reprogramming of NSPCs to rescue neurologic function after injury. In this study, we aimed at defining different subtypes of NSPCs by individual histone methylations. We found the three histone marks, histone H3 lysine 4 trimethylation (H3K4me3), histone H3 lysine 27 trimethylation (H3K27me3), and histone H3 lysine 36 trimethylation (H3K36me3), to nicely and dynamically portray individual cell types during neurodevelopment. First, we found all three marks co-stained with NSPC marker SOX2 in mouse subventricular zone. Then, CD133, Id1, Mash1, and DCX immunostaining were used to define NSPC subtypes. Type E/B, B/C, and C/A cells showed high levels of H3K27me3, H3K36me3, and H3K4me3, respectively. Our results reveal defined histone methylations of NSPC subtypes supporting that epigenetic regulation is critical for neurogenesis and for maintaining NSPCs.
\end{abstract}

Keywords Histone methylation $\cdot$ NSPC subtypes $\cdot$ Mouse subventricular zone $\cdot$ Neurodevelopment

\section{Introduction}

In the postnatal mammalian brain, most of the neural stem/progenitor cells (NSPCs) are spatially restricted to two specific brain regions: the subgranular zone (SGZ) in the dentate gyrus of the hippocampus and the subventricular zone (SVZ)

Electronic supplementary material The online version of this article (https://doi.org/10.1007/s12035-019-01777-5) contains supplementary material, which is available to authorized users.

Magnar Bjørås

magnar.bjoras@ntnu.no

$\bowtie$ Arne Klungland

arne.klungland@medisin.uio.no

1 Institute of Neurobiology, Xi'an Jiaotong University Health Science Center, Xi'an, China

2 Department of Microbiology, Oslo University Hospital, Rikshospitalet, Sognsvannsveien 9, 0372 Oslo, Norway

3 Department of Molecular Medicine, Institute of Basic Medical Sciences, University of Oslo, Oslo, Norway

4 Department of Clinical and Molecular Medicine, Norwegian University of Science and Technology, Høgskoleringen 1, 7491 Trondheim, Norway of the lateral ventricles [1]. As the major site for NSPCs in the postnatal central nervous system (CNS), four major cell types of NSPCs have been identified in the SVZ niche: ependyma-like stem NSPCs (type E cells), quiescent or dormant NSPCs (qNSCs; type B cells), transient amplifying progenitors (TAPs; type $\mathrm{C}$ cells), and migrating neuronal precursors (neuroblasts; type A cells) [2, 3] (Fig. 1b). NSPCs in SVZ can be activated in response to physiological and pathophysiological stimuli, in which they initiate CNS repair and functional recovery [4]. Therefore, understanding the dynamic regulation of NSPC subtypes may provide new insight for developing novel treatment modalities for CNS diseases.

Histone modifications are post-translational modifications to histone proteins which include methylation, phosphorylation, acetylation, ubiquitylation, and sumoylation. These modifications have biological roles and can be inherited and are referred to as epigenetic marks. Specific histone methylation marks at promoter regions affect transcription activities [5]. Generally, histone $\mathrm{H} 3$ lysine 4 trimethylation $(\mathrm{H} 3 \mathrm{~K} 4 \mathrm{me} 3)$ and histone $\mathrm{H} 3$ lysine 36 trimethylation (H3K36me3) are associated with active promoters and gene bodies of actively transcribed genes. This results in increased transcription activity, whereas histone $\mathrm{H} 3$ lysine 27 trimethylation (H3K27me3) is linked to transcriptional 
a

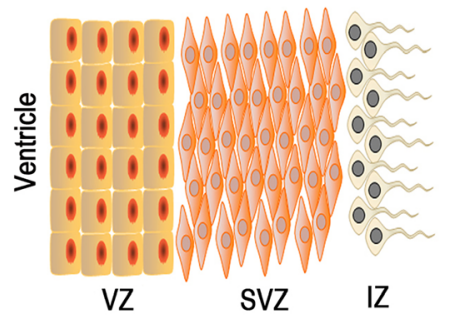

Embryonic mouse brain

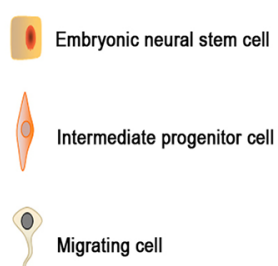

Migrating cell
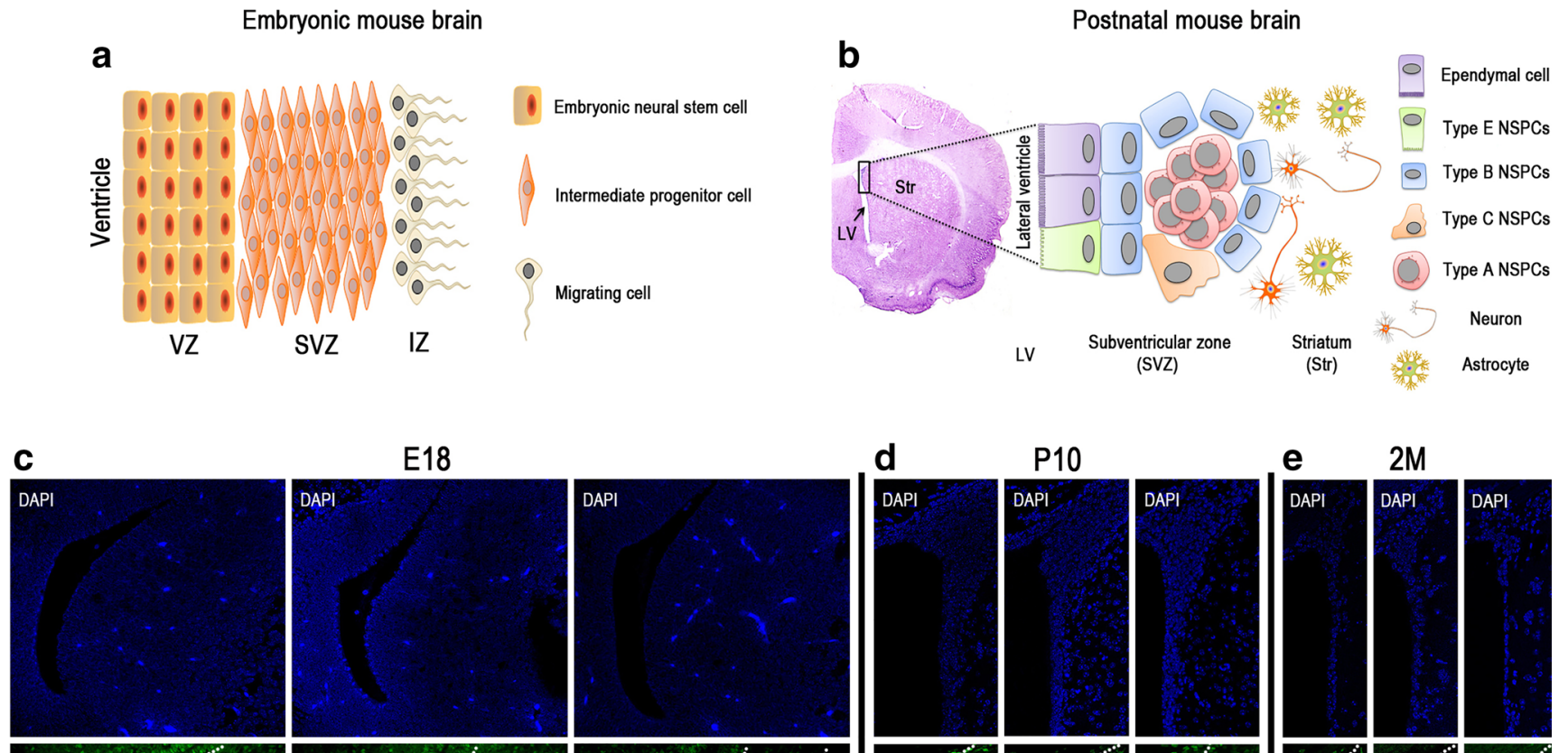

$2 \mathrm{M}$
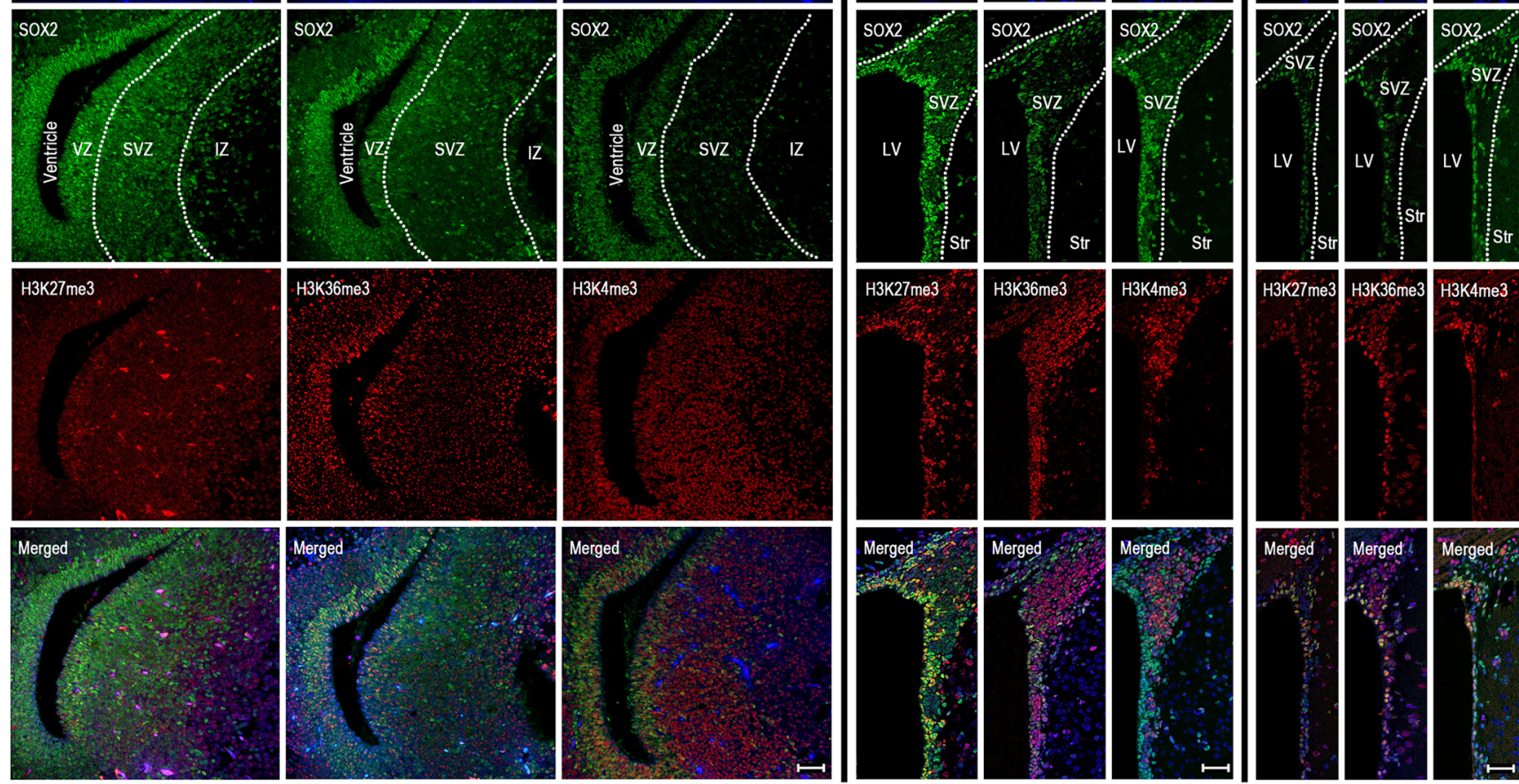

Fig. 1 H3K27me3, H3K36me3, and H3K4me3 co-located with SOX2 during neurodevelopment in SVZ. Schematics of the cell layers and cell types in the embryonic (a) and adult (b) brain. Immunofluorescent staining showed that high level of H3K27me3, H3K36me3, and

H3K4me3 co-stained with SOX2 at E18 (c), P10 (d), and 2M (e). Nuclei were counterstained with DAPI. E18, embryo at day 18; P10, postnatal at day $10 ; 2 \mathrm{M}$, adults 2 months. Scale bar $=50 \mu \mathrm{m}$

repression [6]. H3K4me3, H3K36me3, or H3K27me3 has pivotal and distinct roles in different stages of neurodevelopment and aberrant regulation of histone methylation contributes to the pathogenesis of various CNS disorders [7]. Many embryonic stem cell (ESC) promoters combine activating H3K4me3 marks and repressive $\mathrm{H} 3 \mathrm{~K} 27 \mathrm{me} 3$ marks, and these bivalent domains are important dynamically regulated targets in the expression of developmental genes [8]. H3K36me3 is markedly enriched at pericentromeric heterochromatin in ESCs and fibroblasts
[9]. Even though both H3K4me3 and H3K36me3 are transcriptional activators, $\mathrm{H} 3 \mathrm{~K} 36 \mathrm{me} 3$ predominates in the transcribed bodies of genes, whereas nucleosomes near the transcription start site of active genes contain H3K4me3 [10]. However, we have limited understanding regarding the function of the dynamic changes in these histone methylation marks during neurodevelopment.

In this study, we observed distinct features of histone methylation in the different subtypes of NSPCs during 
neurodevelopment. Type E/B cells are marked by high levels of $\mathrm{H} 3 \mathrm{~K} 27 \mathrm{me} 3$, type $\mathrm{B} / \mathrm{C}$ cells showed high levels of $\mathrm{H} 3 \mathrm{~K} 36 \mathrm{me}$, and $\mathrm{H} 3 \mathrm{~K} 4 \mathrm{me} 3$ is specific for type C/A cells. These results may reveal new insight into the onset of neurodevelopment and provide an innovative epigenetic signature for discovery and characterization of key regulatory genes/regions for neurogenesis.

\section{Material and Methods}

\section{Animals}

C57BL/6N mouse strain was used for this research and all mouse experiments were approved by the Animal Research Committee and the Norwegian Food Safety Authority (NFDA), and conducted in accordance with the rules and regulations of the Federation of European Laboratory Animal Science Associations (FELASA). The staff at Komparativ Medisin (KPM) Oslo University Hospital is responsible for housing and daily maintenance. Housing and environmental enrichment is according to standards. All efforts were made to minimize animal suffering and to keep the numbers of animals used to a minimum.

\section{Method Details}

P10 and adult mice were anesthetized and transcardially perfused with normal saline followed by $4 \%$ paraformaldehyde (PFA, sc-281692, Santa Cruz Biotechnology, Dallas, TX, USA). Ten milliliter normal saline and $25 \mathrm{ml} \mathrm{4 \%} \mathrm{PFA} \mathrm{were}$ used for P10 mice, while adult mice were infused with $25 \mathrm{ml}$ normal saline and $50 \mathrm{ml} \mathrm{4 \%}$ PFA. For E18 mice, pregnant E18 mice were sacrificed and the fetal brains were dissected in cold PBS, and then soaked into 4\% PFA for fixation. All brains were dissected and post-fixed in 4\% PFA overnight at $4{ }^{\circ} \mathrm{C}$, followed by paraffin embedding. Fourmicrometer brain tissue serial slices were coronally sectioned by microtome (HM355s, Thermo Scientific, Waltham, MA, USA) and mounted onto glass slides. These sections were used for immunostaining. The slides were deparaffinized and cleared in Clear-Rite ${ }^{\mathrm{TM}} 3$ (6901TS, Thermo Scientific) followed by rehydration in an EtOH gradient. Then the slides were heated to $95{ }^{\circ} \mathrm{C}$ in the antigen retrieval buffer (3 g sodium citrate (25114, Sigma-Aldrich), $0.4 \mathrm{~g}$ citric acid (251275, Sigma-Aldrich), $1000 \mathrm{~mL} \mathrm{H}_{2} \mathrm{O}$, pH 6.0) for $30 \mathrm{~min}$, followed by washing with $0.01 \mathrm{M}$ PBS (all washes were performed three times, $5 \mathrm{~min}$ each). The slides were permeabilized with $0.3 \%$ Triton X-100 (T8787,

Table 1 Antibodies used during the study

\begin{tabular}{|c|c|c|c|}
\hline Antigen & Source and host species & Concentration & Catalog no. \\
\hline Anti-SOX2 & Abcam, mouse monoclonal antibody & $1: 200$ & ab79351 \\
\hline Anti-SOX2 & Abcam, rabbit polyclonal antibody & $1: 200$ & ab97959 \\
\hline Anti-Ki67 & Abcam, rabbit polyclonal antibody & $1: 200$ & ab15580 \\
\hline Anti-Ki67 & Invitrogen, rat monoclonal antibody & $1: 1000$ & $14-5698-80$ \\
\hline Anti-PCNA antibody & Abcam, rabbit monoclonal antibody & $1: 1000$ & ab29 \\
\hline Anti-PCNA antibody & Abcam, mouse monoclonal antibody & $1: 200$ & ab92552 \\
\hline Anti-CD133 antibody & Millipore, rat monoclonal antibody & $1: 100$ & MAB4310 \\
\hline Anti-ID1 antibody & R\&D Systems, goat polyclonal antibody & $1: 200$ & AF4377 \\
\hline Anti-MASH1 antibody & Abcam, rabbit monoclonal antibody & $1: 1000$ & $\mathrm{ab} 213151$ \\
\hline Anti-MASH1 antibody & BD Pharmingen, mouse monoclonal antibody & $1: 100$ & 556604 \\
\hline Anti-DCX antibody & Abcam, rabbit polyclonal antibody & $1: 200$ & ab18723 \\
\hline Anti-DCX antibody & $\begin{array}{l}\text { BD Transduction Laboratories, mouse monoclonal } \\
\text { antibody }\end{array}$ & $1: 200$ & 611706 \\
\hline Anti-trimethyl-Histone H3 (Lys4) (H3K4me3) antibody & Millipore, rabbit monoclonal antibody & $1: 500$ & $04-745$ \\
\hline Anti-Histone H3 trimethyl Lys36 (h3k36me3) antibody & Active Motif, mouse monoclonal antibody & $1: 500$ & 61021 \\
\hline Anti-Histone h3k27me3 (H3K27 Trimethyl) antibody & Epigentek, rabbit Polyclonal antibody & $1: 150$ & A-4039 \\
\hline Anti-Mouse igg $(\mathrm{H}+\mathrm{L})$ secondary antibody, Alexa Fluor 488 & Invitrogen, donkey polyclonal antibody & $1: 500$ & R37114 \\
\hline Anti-Mouse $\operatorname{IgG}(\mathrm{H}+\mathrm{L})$ secondary antibody, Alexa Fluor 594 & Invitrogen, donkey polyclonal antibody & $1: 500$ & R37115 \\
\hline Anti-Rabbit IgG $(\mathrm{H}+\mathrm{L})$ secondary antibody, Alexa Fluor 488 & Invitrogen, donkey polyclonal antibody & $1: 500$ & R37118 \\
\hline Anti-Rabbit IgG (H+L) secondary antibody, Alexa Fluor 594 & Invitrogen, donkey polyclonal antibody & $1: 500$ & R37119 \\
\hline $\begin{array}{l}\text { Anti-Rat IgG }(\mathrm{H}+\mathrm{L}) \text { Highly Cross-Adsorbed secondary antibody, } \\
\text { Alexa Fluor } 594\end{array}$ & Invitrogen, donkey polyclonal antibody & $1: 500$ & A-21209 \\
\hline $\begin{array}{l}\text { Anti-Goat IgG }(\mathrm{H}+\mathrm{L}) \text { Cross-Adsorbed secondary antibody, Alexa } \\
\text { Fluor } 594\end{array}$ & Invitrogen, donkey polyclonal antibody & $1: 500$ & A-11058 \\
\hline
\end{tabular}



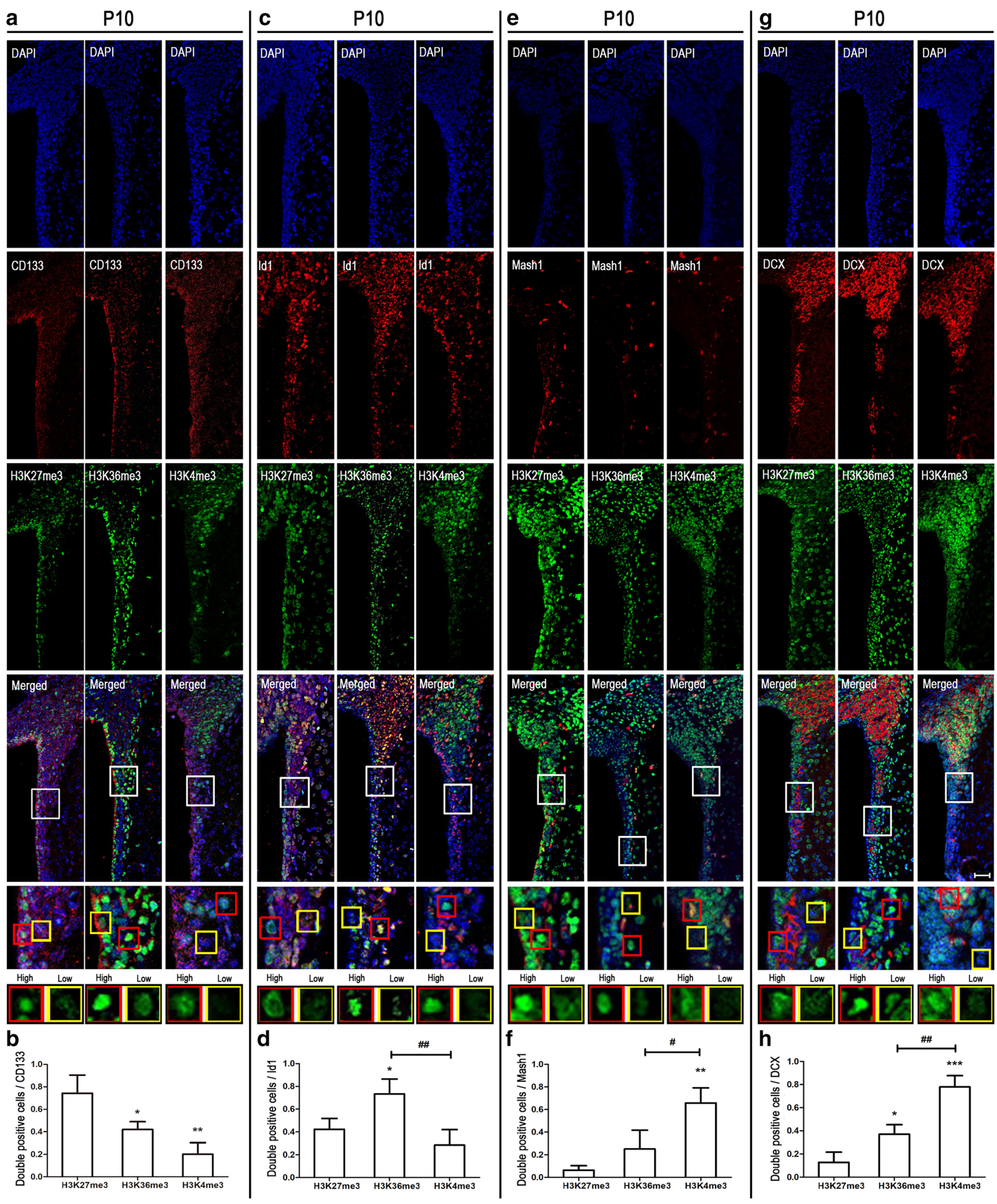

Fig. 2 Different subtypes of NSPC marker co-stained with different histone methylations at postnatal 10 days (P10). Immunocytochemical double labeling revealed different patterns of histone methylations costained with CD133 (a), Id1 (c), Mash1 (e), and DCX (g) respectively. Nuclei were counterstained with DAPI. Scale bar $=50 \mu \mathrm{m}$. The square frames are enlarged to identify a single typical high (red) and low (yellow)

level cell relating to the different histone methylation mark. $\mathbf{b}, \mathbf{d}, \mathbf{f}, \mathbf{h}$ The number of immunolabeled cells was counted for three sections in each mouse and each value represents the mean $\pm \mathrm{SD}$ of three mice $(n=3)$. $* P$ $<0.05, * * P<0.01, * * * P<0.001$ versus $\mathrm{H} 3 \mathrm{~K} 27 \mathrm{me} 3$ group; ${ }^{\#} P<0.05$, ${ }^{\# \#} P<0.01$ versus $\mathrm{H} 3 \mathrm{~K} 36 \mathrm{me} 3$ group. $\mathrm{P} 10$, postnatal at day 10 

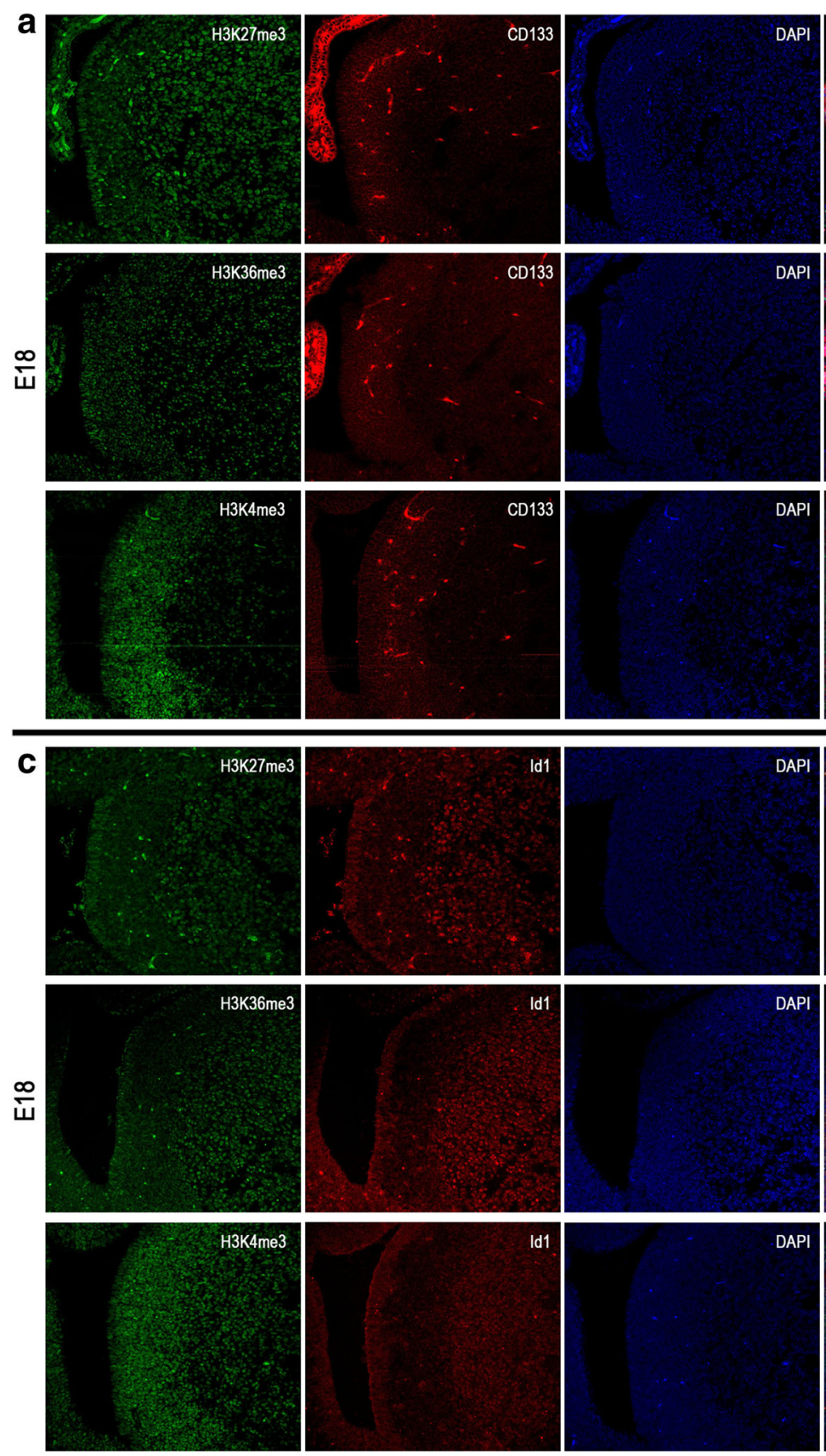

Fig. 3 CD133 and Id1 co-stained with different histone methylations at E18. Immunocytochemical double labeling revealed different patterns of histone methylations co-stained with CD133 (a) and Id1 (c), respectively. Nuclei were counterstained with DAPI. Scale bar $=50 \mu \mathrm{m}$. b, d The

Sigma-Aldrich, St. Louis, MO, USA) for $20 \mathrm{~min}$, rinsed, and then blocked for $2 \mathrm{~h}$ with blocking buffer (5\% normal goat serum (G9023, Sigma-Aldrich) and 5\% bovine serum albumin (A7096, Sigma-Aldrich)). The samples were incubated with the primary antibodies (Table 1) overnight at $4{ }^{\circ} \mathrm{C}$, washed with $0.01 \mathrm{M} \mathrm{PBS}$, and then incubated with the suitable secondary antibodies. All antibodies are shown in Key resources table. The control samples were incubated in
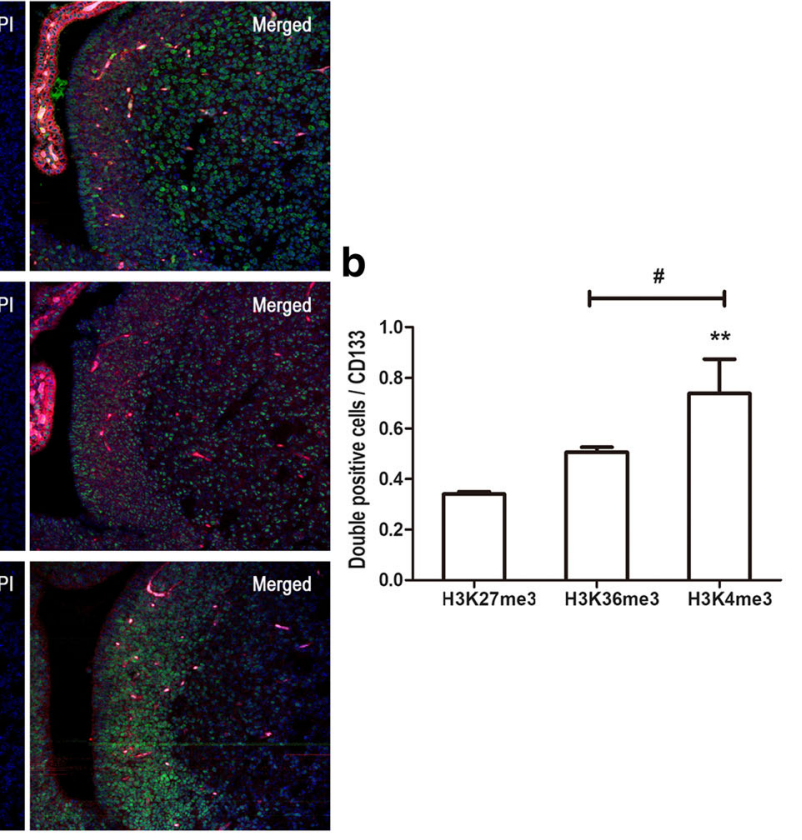

DAPI
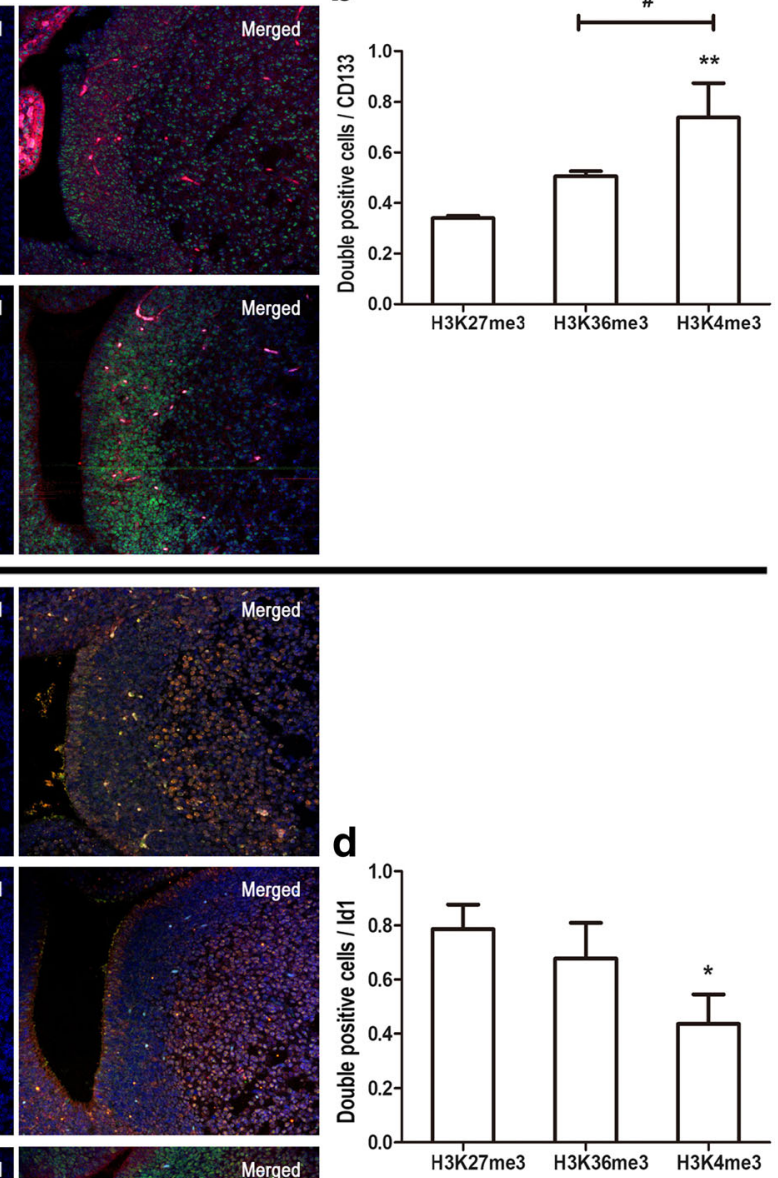

DAPI

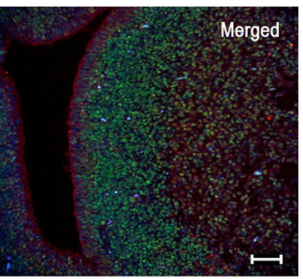

number of immunolabeled cells was counted for three sections in each mouse and each value represents the mean $\pm \mathrm{SD}$ of three mice $(n=3)$. $* P$ $<0.05, * * P<0.01$ versus $\mathrm{H} 3 \mathrm{~K} 27 \mathrm{me} 3$ group; $\# P<0.05$ versus H3K36me3 group. E18, embryo at day 18

blocking buffer instead without primary antibodies. Nuclei were visualized with mounting medium including DAPI (H-1200, Vector, Burlingame, CA, USA). Images were taken with a Leica SP8 confocal microscope equipped with a $\times 40$ oil immersion lens. The number of immunolabeled cells lining the lateral wall of the lateral ventricle was counted for three sections in each mouse, and at least three animals were used for each experiment. 


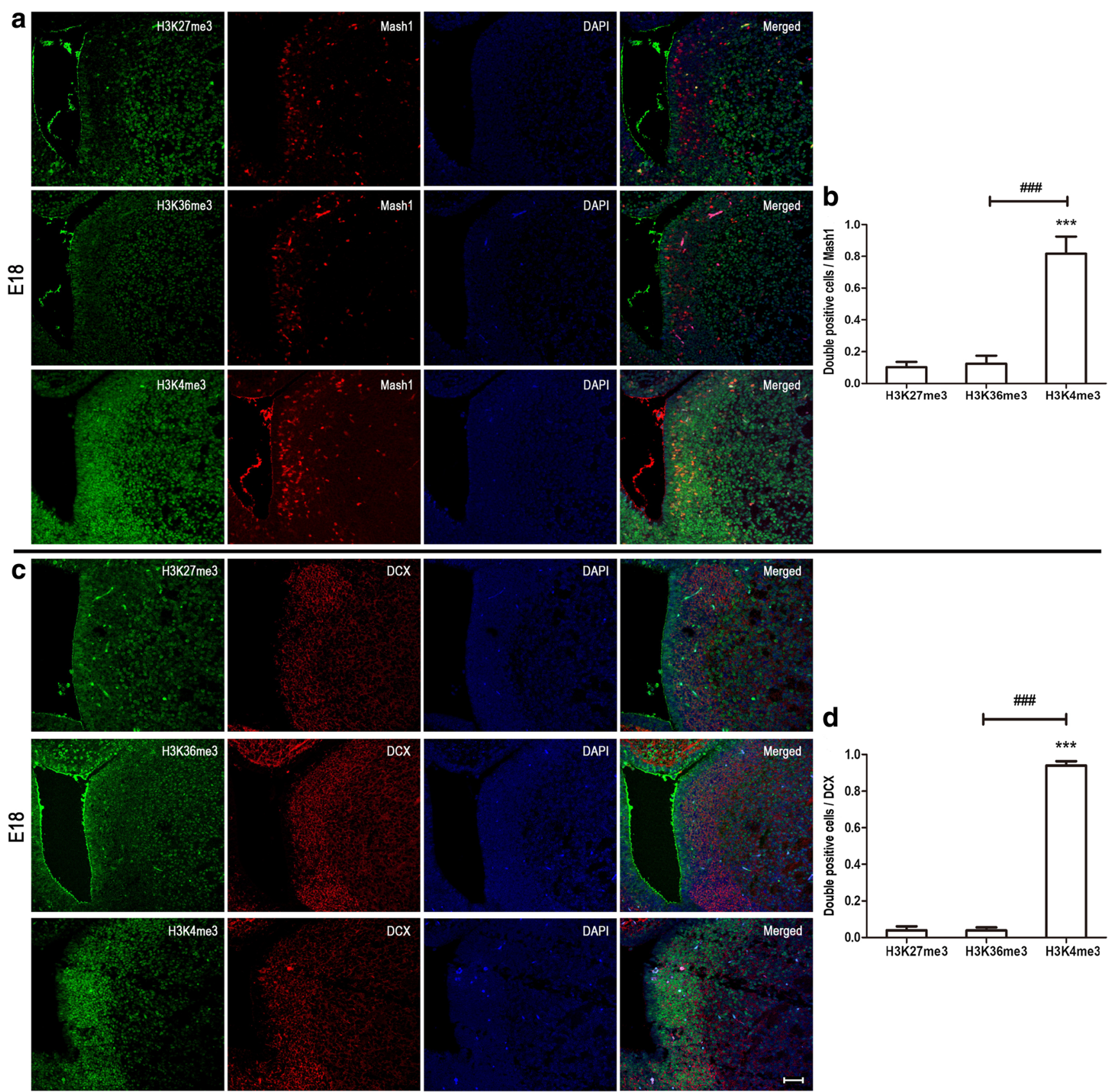

Fig. 4 Mash1 and DCX co-stained with different histone methylations at E18. Immunocytochemical double labeling showed different patterns of histone methylations in Mash1- (a) and DCX (c)-positive cells. Nuclei were counterstained with DAPI. Scale bar $=50 \mu \mathrm{m}$. b, d The number of

\section{Quantification and Statistical Analysis}

The level of histone methylation and double-positive cell was measured and defined by using Image-Pro Plus 5.1. Differences between groups were analyzed using one-way ANOVA, followed by Tukey's post hoc test. All statistical analyses were performed using GraphPad Prism 5. The data are shown as mean \pm standard deviation, and $P<0.05$ was considered as statistically significant difference. immunolabeled cells was counted for three sections in each mouse and each value represents the mean $\pm \mathrm{SD}$ of three mice $(n=3)$. *** $P<0.001$ versus H3K27me3 group; \#\#\#P<0.001 versus H3K36me3 group. E18, embryo at day 18

\section{Results}

High Levels of H3K27me3, H3K36me3, and H3K4me3 in Neural Stem/Precursor Cells during Neurodevelopment in SVZ

To characterize the dynamics of histone methylations during neurodevelopment, we collected mouse brains at different time points of early life: embryo at day 18 (E18), postnatal at day 10 


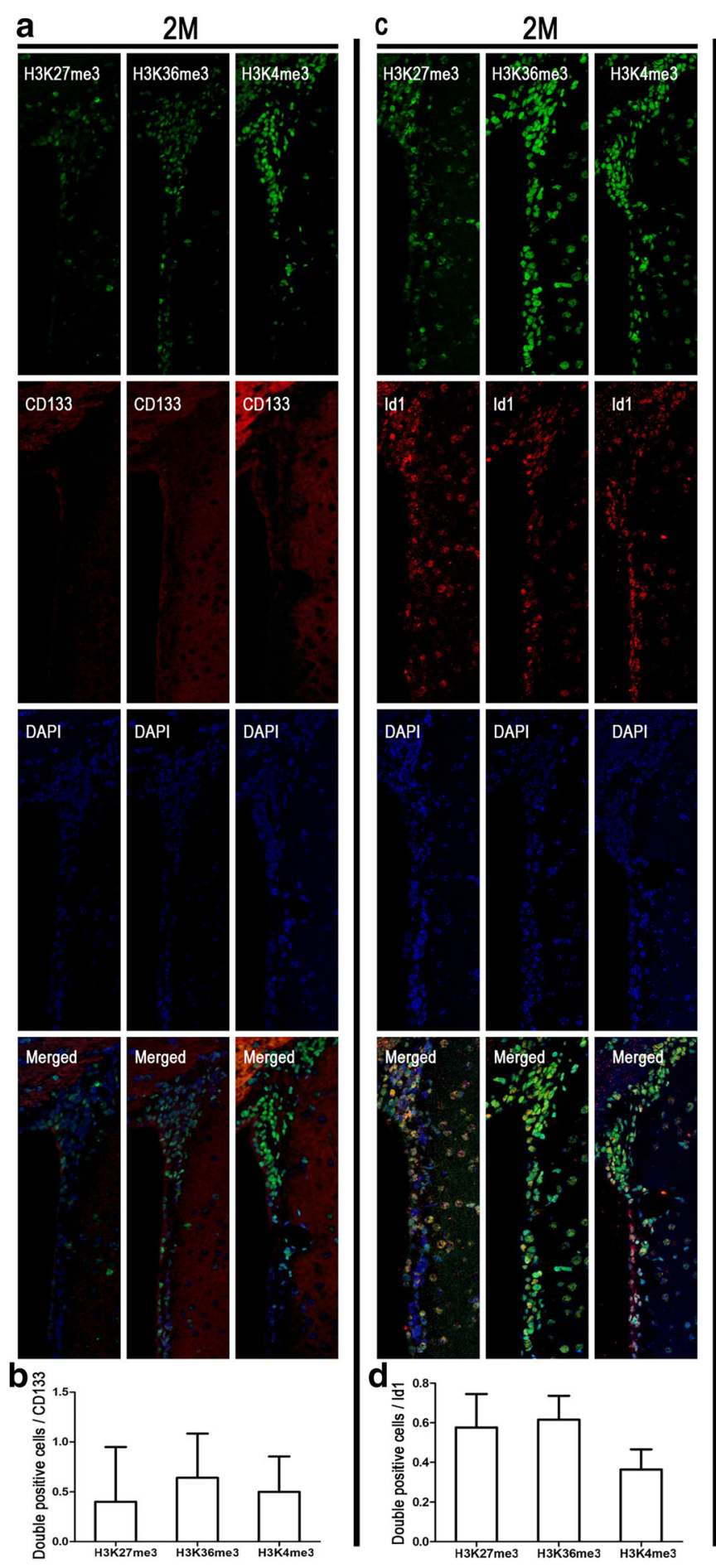

Fig. 5 Different subtypes of NSPC marker co-stained with different histone methylations at 2 months. Immunocytochemical double labeling showed different patterns of histone methylations co-stained with CD133 (a), Id1 (c), Mash1 (e), and DCX (g), respectively. Nuclei were

(P10), and adults at 2 months $(2 \mathrm{M})$. Then, we examined the levels of three different histone methylation marks (H3K27me3, H3K36me3, and H3K4me3) by immunofluorescence staining. All three histone marks showed strongest staining in neurogenic niches (e.g., SVZ and SGZ) during

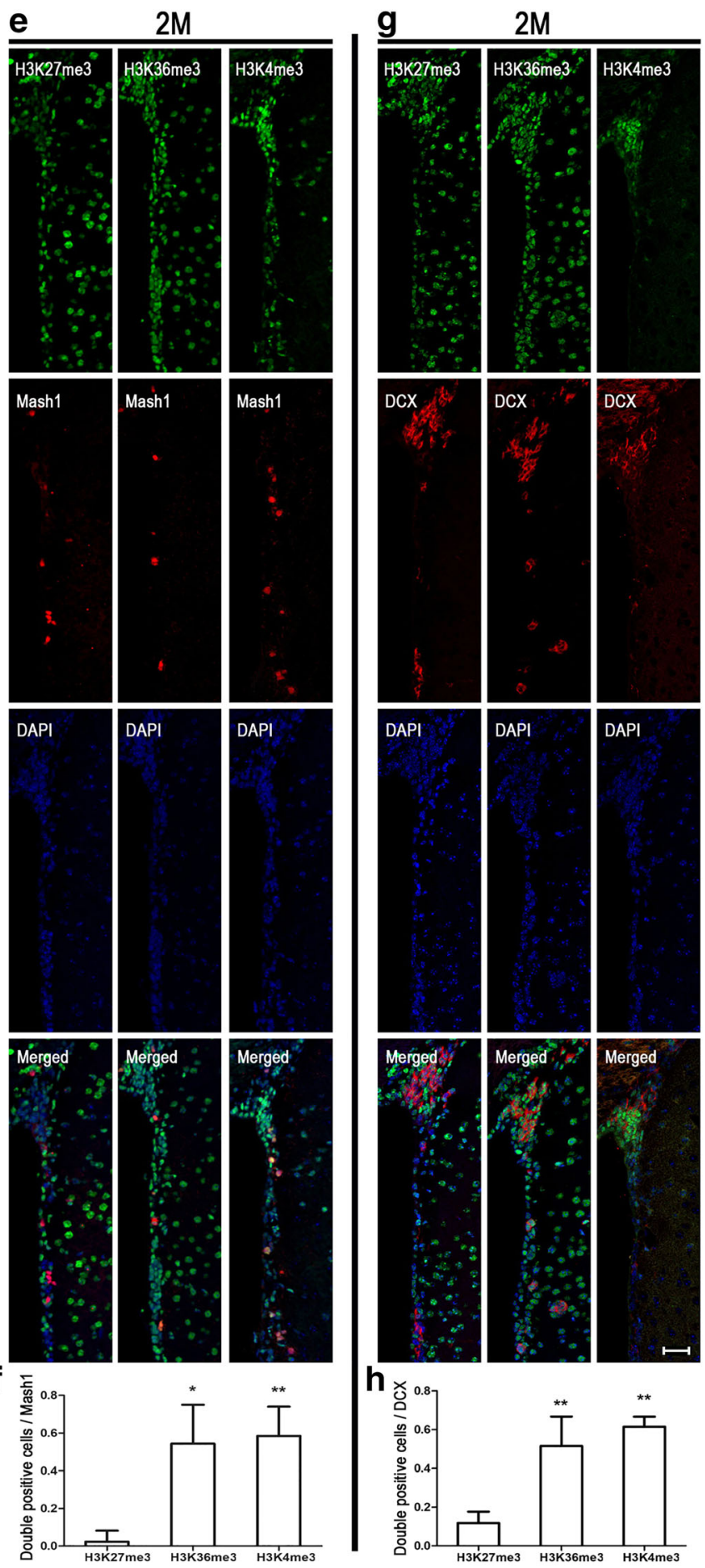

counterstained with DAPI. Scale bar $=50 \mu \mathrm{m}$. b, d, f, and $\mathbf{g}$ The number of immunolabeled cells was counted for three sections in each mouse and each value represents the mean $\pm \mathrm{SD}$ of three mice $(n=3)$. $* P$ $<0.05, * * P<0.01$ versus H3K27me3 group. 2M, 2 months

neurodevelopment, although the intensity varied among the three time points. In SVZ, the three tested histone marks showed co-localization with the established NSPC marker SOX2 at all three time points of development studied (Fig. 1c-e). In SGZ, SOX2 co-localized with H3K4me3 and H3K36me3 at all three 


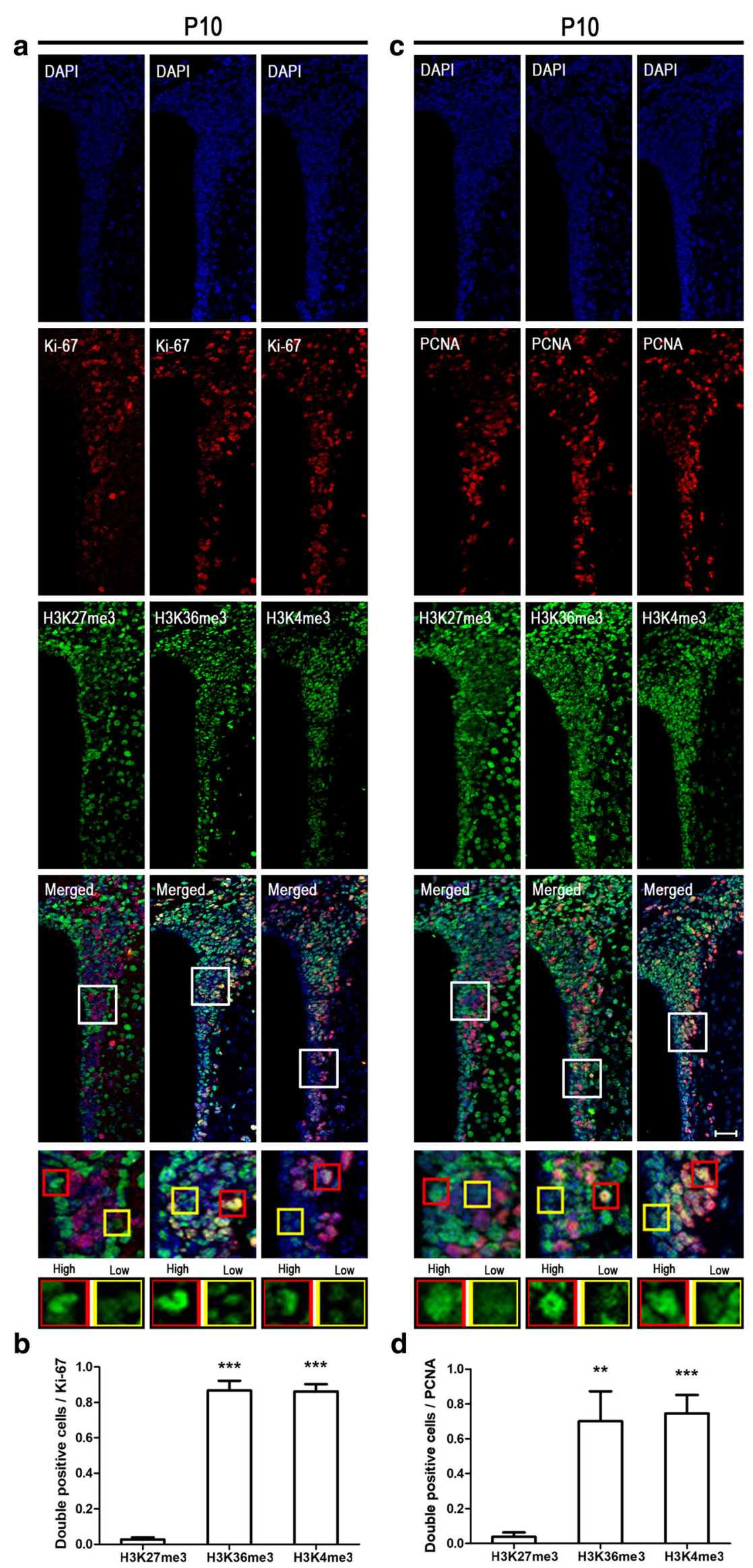


Fig. 6 High levels of $\mathrm{H} 3 \mathrm{~K} 4 \mathrm{me} 3$ and $\mathrm{H} 3 \mathrm{~K} 36 \mathrm{me} 3$ in cells expressing Ki67 and PCNA at postnatal 10 days (P10). Immunocytochemical double labeling showed different patterns of histone methylations in Ki-67- (a) and PCNA (c)-positive cells. Nuclei were counterstained with DAPI. Scale bar $=50 \mu \mathrm{m}$. The square frames are enlarged to show the typical detail high (red) and low (yellow) levels of different histone methylation features. b, d The number of immunolabeled cells was counted for three sections in each mouse and each value represents the mean $\pm \mathrm{SD}$ of three mice $(n=3)$. $* * P<0.01$, $* * * P<0.001$ versus H3K27me3 group. P10, postnatal at day 10

time points, while H3K27me3 SOX2 double-positive cells were just sporadic at any time point (Supplement Fig. 1 A-D). Notably, H3K4me3, H3K36me3, and H3K27me3 stained distinct parts of SVZ, particularly at P10. H3K27me3 showed strongest staining of the ependymal cell layer, and H3K36me3 level was high in the surrounding striatal parenchyma as well as the ependymal cell layer at the lateral ventricle. In contrast, $\mathrm{H} 3 \mathrm{~K} 4 \mathrm{me} 3$ staining is strongest between the ependymal cell layer and the striatal parenchyma (Fig. 1d). Previously, it has been demonstrated that in the postnatal mouse brain, type B cells locate between type A cells and the underlying striatal parenchyma as well as between type A cells and the ependymal cells, and that type $\mathrm{C}$ cells locate around type A cells (Fig. 1b) [11, 12]. These results suggest that histone methylation may define different subtypes of NSPCs.

\section{High Level of H3K27me3 in CD133-Positive Cells at Early Postnatal Neurodevelopment}

In postnatal mouse brain, CD133 (also known as prominin-1) is a marker for type $\mathrm{E} / \mathrm{B}$ cells; $\mathrm{Id} 1$ marks type $\mathrm{B} / \mathrm{C}$ cells (type $\mathrm{C}$ cells are Id1 positive, although at significantly lower levels relative to type $\mathrm{B}$ cells) [13]; type $\mathrm{C}$ cells express the highest levels of Mash1 (also known as Ascl1); and DCX marks type A cells [14]. Immunocytochemical double labeling unveils 74\% CD133-positive cells showing high level of $\mathrm{H} 3 \mathrm{~K} 27 \mathrm{me} 3$. On the contrary, there were few CD133positive cells that co-stained with H3K36me3 (42\%) and H3K4me3 (20\%) (Fig. 2a, b). In the adult SVZ, the number of CD133-positive cell decreased markedly and there was no significant difference in immunocytochemical double labeling for the three histone methylation marks studied at this stage (Fig. 5a, b). The anatomical structure of embryo and postnatal mouse brain is noticeably different (Fig. 1a, b). In embryo mouse brain, H3K27me3 and H3K36me3 showed high levels in the ventricular zone (VZ), while high levels of H3K4me3 cells were located to SVZ (Fig. 1c). As expected, most cells in $\mathrm{VZ}$ and SVZ were CD133 positive. Furthermore, $74 \%$ of the CD133-positive cells co-stained with $\mathrm{H} 3 \mathrm{~K} 4 \mathrm{me} 3$, while H3K27me 3 and H3K36me 3 showed $34 \%$ and 51\% costaining with CD133, respectively (Fig. 3a, b). Thus, it appears that high level of $\mathrm{H} 3 \mathrm{~K} 27 \mathrm{me} 3$ is displayed in ependymal and quiescent neural stem cells in the SVZ (type B/E) at early postnatal neurodevelopment.

\section{High Level of H3K36me3 in Id1-Positive Cells at Early Postnatal Neurodevelopment}

Similarly, we used immunocytochemical double labeling to identify colocalization of Id 1 and the three histone methylation marks. Seventy-three percent Id1-positive cells co-stained with H3K36me3, significantly higher than H3K27me3 (42\%) and H3K4me3 (29\%) (Fig. 2c, d). Analogous to the CD133 staining, the number of Id1-positive cells was reduced dramatically at adulthood and double labeling revealed minor differences for the three histone methylation marks (Fig. 5c, d). In embryo mouse brain, most of the Id1-positive cells were located in $\mathrm{VZ}$ and the majority of Id1-positive cells co-stained with H3K27me3 (79\%) and H3K36me3 (68\%) (Fig. 3c, d). However, just 44\% H3K4me3-positive cells co-stained with Id1. These phenomena may indicate that $\mathrm{H} 3 \mathrm{~K} 36 \mathrm{me} 3$ is a good marker for quiescent and active neural stem cells (type $\mathrm{B} / \mathrm{C}$ ) at early postnatal neurodevelopment.

\section{High Level of H3K4me3 in Mash1 and DCX-Positive Cells at Postnatal Neurodevelopment}

Mash1 (also known as Ascl1) is characterized as a proneural transcription factor and typically used as a type $\mathrm{C}$ cell marker. DCX is expressed in the last stage before NSPCs are migrating through the rostral migratory stream (RMS) [14]. Therefore, Mash1 and DCX were used for labeling type C and A cell, respectively. Immunocytochemical double labeling identified $66 \%$ of Mash1-positive cells co-staining with $\mathrm{H} 3 \mathrm{~K} 4 \mathrm{me} 3$ at $\mathrm{P} 10$, while very low co-staining was observed for H3K27me3 (6\%) and H3K36me3 (25\%) (Fig. 2 E, F). Embryonic brain staining results showed that Mash1-positive cell appeared in SVZ; and it was similar to P10 with $82 \% \mathrm{H} 3 \mathrm{~K} 4 \mathrm{me} 3$, and very low H3K27me3 and H3K36me3 co-staining, 10\% and 13\% respectively (Fig. 4a, b). At adulthood, 58\% Mash1-positive cells co-stained with $\mathrm{H} 3 \mathrm{~K} 4 \mathrm{me} 3,2 \%$ with $\mathrm{H} 3 \mathrm{~K} 27 \mathrm{me} 3$, and $54 \%$ with H3K36me3 (Fig. 5e, f). Then, double immunostaining was also used for detecting DCX and different histone methylations. During neurodevelopment, the number of H3K4me3 DCX double-positive cells was significantly higher compared with H3K27me3 or H3K36me3 double-positive cells (Fig. 2g, h; Fig. 4c, d; and Fig. 5g, h). Thus, both type $\mathrm{C}$ and type $\mathrm{A}$ are represented by $\mathrm{H} 3 \mathrm{~K} 4 \mathrm{me} 3$.

\section{H3K4me3- and H3K36me-Positive Cells Co-Stain with Proliferation Markers at Early Neurodevelopment}

To further evaluate histone methylation in the proliferation state of early developmental cells in SVZ, co-staining with 
the proliferation markers Ki-67 and PCNA was analyzed. We identified a noticeable difference with most of $\mathrm{Ki}$-67-positive cells co-staining with H3K36me3 (87\%) or H3K4me3 (86\%), while only $3 \%$ of H3K27me3-positive cells co-stained with Ki-67 at P10 (Fig. 6a, b). Similarly, just 4\% of PCNA-positive cells co-stained with $\mathrm{H} 3 \mathrm{~K} 27 \mathrm{me} 3$ compared with $70 \%$ for H3K36me3 and 75\% for H3K4me3 (Fig. 6c, d). These results strongly indicate that high levels of H3K $36 \mathrm{me} 3$ and H3K 4em3 correlate very well with proliferating cells in SVZ at early postnatal neurodevelopment.

\section{Discussion}

Traditional therapies for CNS diseases are limited. For example, treatment for clinical stroke by the administration of tissue plasminogen activator and the recent introduction of mechanical thrombectomy can only be used in a limited proportion of patients due to time constraints [15]. Accordingly, continuing efforts are in need to develop novel, safe, and more optimal and effective therapeutic strategies for CNS diseases. The dynamic regulation of histone methylations and chromatin remodeling plays essential roles in development, cellular differentiation, and cell fate maintenance [16]. More importantly, emerging evidence supports the involvement of histone methylation in the pathogenesis of CNS damage and several neurodegenerative diseases $[17,18]$. In this study, we reveal how different histone methylation marks are dynamically regulated during NSPC differentiation in the mouse SVZ area, represented as marked differences in histone methylations between quiescent and active NSPCs (Fig. 7). As NSPCs can be activated by CNS damage and participate in CNS repair and functional recovery, our study may bring a novel perspective to a therapeutic strategy of CNS diseases and provide a potential histone methylation features for screening and identifying key therapeutic genes for CNS diseases.

SOX2 maintain stemness of NSPCs in a slowly proliferating stem cell state by repressing the cell cycle regulator cyclin D1 during cortex development [19]. When NSPCs enter the stage of differentiation, the levels of SOX2 decrease, which releases this repression and thus promotes cell cycle re-entry and NPC proliferation [20]. In this study, the SOX2 staining results showed that the number of SOX2 cells in SVZ gradually decreased during neurodevelopment. Notably, most cells with high level of H3K27me3 showed high level of SOX2, whereas H3K36me3 cells presented low co-staining with SOX2 cells. Furthermore, H3K4me3 and SOX2 co-staining are rare in SVZ. There is a positive correlation between the expression of SOX2 and stemness of NSPCs [21]. Thus, our results define histone methylations specific for SOX2-positive NSPCs. Moreover, we reveal that high levels of H3K27me3 exist in the early stage of NSPC development; H3K36me3 is characteristic of metaphase while $\mathrm{H} 3 \mathrm{~K} 4 \mathrm{me} 3$ is enriched in the mid and later stages of NSPC development.

In the mammalian embryo brain, the proliferative region comprises two distinct zones: VZ, which is a neuroepithelial layer directly adjacent to the ventricular lumen, and SVZ, which is positioned superficial to the ventricular zone [22] (Fig. 1a). Radial glial cells (RGCs, one type of embryonic neural stem cells) reside in the VZ and generate both intermediate progenitor cells (IPCs, one type of embryonic neural precursor cells) and cortical neurons. IPCs migrate away from
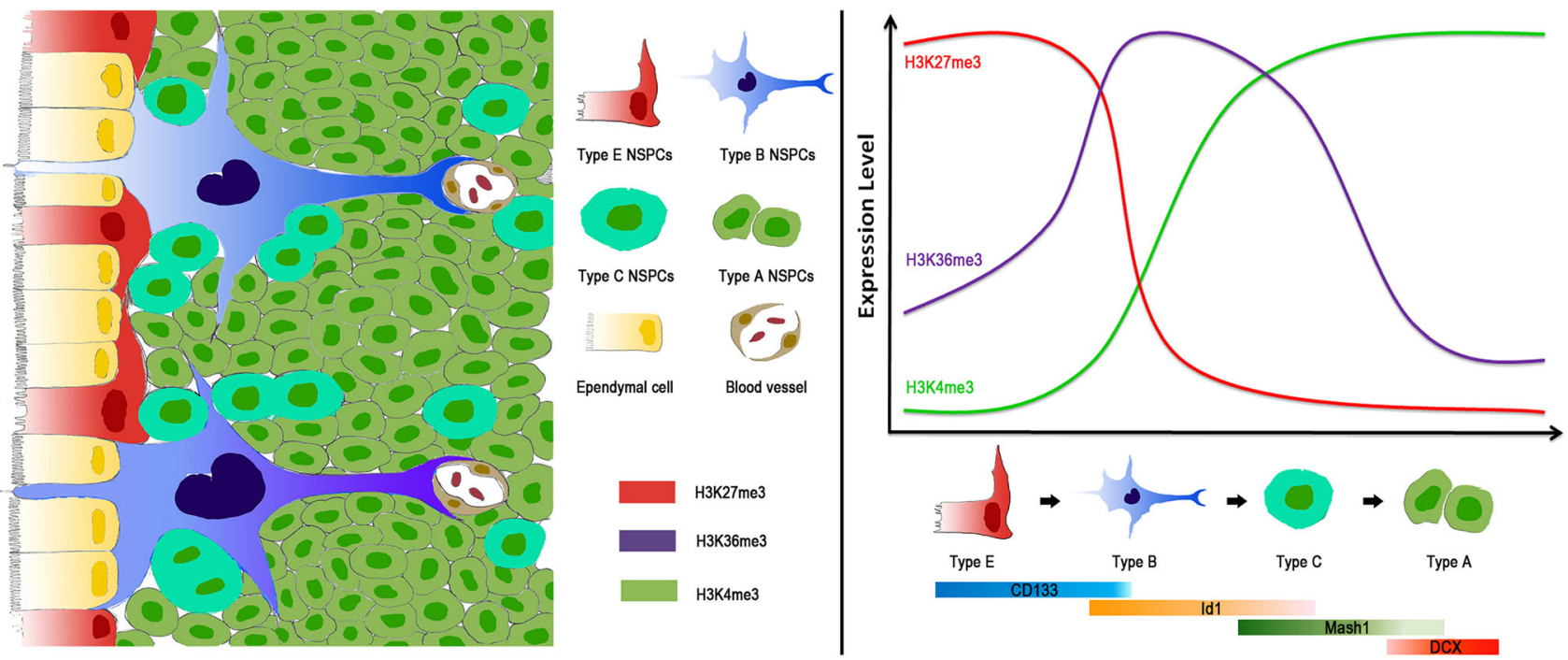

Fig. 7 Schematic model of the developmental process of NSPCs projected from this study. Histone methylations are dynamically changed during NSPC differentiation in mouse SVZ area. Different subtypes of NSPCs represented different patterns of histone methylations. Specifically, type E/B cells are marked by high levels of $\mathrm{H} 3 \mathrm{~K} 27 \mathrm{me} 3$, type B/C cells showed high levels of H3K36me3, and $\mathrm{H} 3 \mathrm{~K} 4 \mathrm{me} 3$ is specific for type C/A cells 
the ventricular surface and establish the SVZ [23]. Therefore, the cellular composition is different in VZ and SVZ. RGCs are mostly concentrated in VZ and most of IPCs located in SVZ. In this study, embryo brain staining showed high levels of $\mathrm{H} 3 \mathrm{~K} 27 \mathrm{me} 3$ and $\mathrm{H} 3 \mathrm{~K} 36 \mathrm{me} 3$ in $\mathrm{VZ}$, and of $\mathrm{H} 3 \mathrm{~K} 4 \mathrm{me} 3$ in SVZ. Thus, it suggests high level of H3K27me3 and H3K36me3 at early stage of embryonic neural stem cell development, and of $\mathrm{H} 3 \mathrm{~K} 4 \mathrm{me} 3$ at middle/late stage.

Further, our results identify significant differences among immunocytochemical double labeling in the P10 SVZ. However, we found that these distinct features disappeared in 2 months or E18; the number of NSPCs in SVZ was significantly decreased during neuronal development, and the dynamics of histone methylations described here might be one of the mechanisms underlying this regulation and might encode the difference between embryonic NSPCs and adult NSPCs. One major difference between adult and embryonic neural stem cells is their different number and their ability to differentiate into various cell types. Embryonic NSPCs can divide asymmetrically to generate neurons directly or indirectly through intermediate progenitor cells and oligodendrocytes. More importantly, at the end of the embryonic development, embryonic NSPCs begin to detach from the apical side and convert into astrocytes. Even if adult NSPCs can continue to generate neurons and oligodendrocytes, they cannot differentiate into astrocytes [24]. Histone methylation introduces epigenetic modifications with close ties to transcription and has been directly linked to lifespan regulation in many organisms [25]. For example, upon differentiation towards the neuronal lineage, some bivalent genes became expressed and lost the H3K27me3 mark, whereas those that were silenced lost the $\mathrm{H} 3 \mathrm{~K} 4 \mathrm{me} 3$ and retained H3K27me3 [26]. Therefore, it is not unlikely that the embryonic and adult NSPC states are maintained by differential histone methylation profiles.

Chromatin, the template for epigenetic regulation, is a highly dynamic entity that is constantly reshaped during neurodevelopment [27]. Epigenetic regulation by histone methylation provides the necessary plasticity for cells to respond to environmental and positional cues, enabling the maintenance of acquired information without changing the DNA sequence. In this study, we showed different subtypes of NSPCs represented different features of histone methylations. These results may reveal novel insight into the onset of neurodevelopment and provide an innovative epigenetic signature for discovery and characterization of key regulatory genes for neurogenesis. However, further studies, especially whole epigenome analysis and histone profiling, are necessary for in-depth understanding of the role for individual histone methylation domains in neurodevelopment.

Acknowledgments We thank the animal facility at Oslo University Hospital for mouse handling.
Funding Information This work was funded by the Norwegian Cancer Society (to A.K. and M.B.), the Norwegian Research council, the Health Authority South East, China Postdoctoral Science Foundation (No. 2017M623152, 2018T111035, to Z.Z.) and National Natural Science Foundation of China (No. 81901156, to Z.Z.).

\section{Compliance with Ethical Standards}

Conflict of Interest The authors declare that they have no conflict of interest.

Open Access This article is distributed under the terms of the Creative Commons Attribution 4.0 International License (http:// creativecommons.org/licenses/by/4.0/), which permits unrestricted use, distribution, and reproduction in any medium, provided you give appropriate credit to the original author(s) and the source, provide a link to the Creative Commons license, and indicate if changes were made.

\section{References}

1. Ming GL, Song HJ (2011) Adult neurogenesis in the mammalian brain: significant answers and significant questions. Neuron 70(4): 687-702. https://doi.org/10.1016/j.neuron.2011.05.001

2. Zhao CM, Deng W, Gage FH (2008) Mechanisms and functional implications of adult neurogenesis. Cell 132(4):645-660. https:// doi.org/10.1016/j.cell.2008.01.033

3. Coskun V, Wu H, Blanchi B, Tsao S, Kim K, Zhao J, Biancotti JC, Hutnick L et al (2008) CD133+ neural stem cells in the ependyma of mammalian postnatal forebrain. Proc Natl Acad Sci U S A 105(3):1026-1031. https://doi.org/10.1073/pnas.0710000105

4. Ma DK, Bonaguidi MA, Ming GL, Song HJ (2009) Adult neural stem cells in the mammalian central nervous system. Cell Res 19(6):672-682. https://doi.org/10.1038/cr.2009.56

5. Pedersen MT, Helin K (2010) Histone demethylases in development and disease. Trends Cell Biol 20(11):662-671. https://doi. org/10.1016/j.tcb.2010.08.011

6. Tessarz P, Kouzarides T (2014) Histone core modifications regulating nucleosome structure and dynamics. Nat Rev Mol Cell Biol 15(11):703-708. https://doi.org/10.1038/nrm3890

7. Urdinguio RG, Sanchez-Mut JV, Esteller M (2009) Epigenetic mechanisms in neurological diseases: genes, syndromes, and therapies. Lancet Neurol 8(11):1056-1072. https://doi.org/10.1016/ S1474-4422(09)70262-5

8. Voigt P, Tee WW, Reinberg D (2013) A double take on bivalent promoters. Genes Dev 27(12):1318-1338. https://doi.org/10.1101/ gad.219626.113

9. Chantalat S, Depaux A, Hery P, Barral S, Thuret JY, Dimitrov S, Gerard M (2011) Histone H3 trimethylation at lysine 36 is associated with constitutive and facultative heterochromatin. Genome Res 21(9):1426-1437. https://doi.org/10.1101/gr.118091.110

10. Abed JA, Jones RS (2012) H3K36me3 key to polycomb-mediated gene silencing in lineage specification. Nat Struct Mol Biol 19(12): 1214-1215. https://doi.org/10.1038/nsmb.2458

11. Ming GL, Song H (2005) Adult neurogenesis in the mammalian central nervous system. Annu Rev Neurosci 28:223-250. https:// doi.org/10.1146/annurev.neuro.28.051804.101459

12. Wang YZ, Plane JM, Jiang P, Zhou CJ, Deng W (2011) Concise review: quiescent and active states of endogenous adult neural stem cells: identification and characterization. Stem Cells 29(6):907912. https://doi.org/10.1002/stem.644 
13. Nam HS, Benezra R (2009) High levels of Id 1 expression define B1 type adult neural stem cells. Cell Stem Cell 5(5):515-526. https:// doi.org/10.1016/j.stem.2009.08.017

14. Mamber C, Kozareva DA, Kamphuis W, Hol EM (2013) Shades of gray: the delineation of marker expression within the adult rodent subventricular zone. Prog Neurobiol 111:1-16. https://doi.org/10. 1016/j.pneurobio.2013.07.003

15. Yong-Quan G, Lim YA, Sobey CG, Dheen T, Fann DYW, Arumugam TV (2018) Epigenetic regulation of inflammation in stroke. Ther Adv Neurol Diso 11:175628641877181510.1177/ 1756286418771815

16. Greer EL, Shi Y (2012) Histone methylation: a dynamic mark in health, disease and inheritance. Nat Rev Genet 13(5):343-357. https://doi.org/10.1038/nrg3173

17. Mehler MF (2008) Epigenetic principles and mechanisms underlying nervous system functions in health and disease. Prog Neurobiol 86(4):305-341. https://doi.org/10.1016/j.pneurobio.2008.10.001

18. Berson A, Nativio R, Berger SL, Bonini NM (2018) Epigenetic regulation in neurodegenerative diseases. Trends Neurosci 41(9): 587-598. https://doi.org/10.1016/j.tins.2018.05.005

19. Hagey DW, Klum S, Kurtsdotter I, Zaouter C, Topcic D, Andersson O, Bergsland M, Muhr J (2018) SOX2 regulates common and specific stem cell features in the CNS and endoderm derived organs. PLoS Genet 14(2):e1007224. https://doi.org/10.1371/journal.pgen. 1007224

20. Hagey DW, Muhr J (2014) Sox2 acts in a dose-dependent fashion to regulate proliferation of cortical progenitors. Cell Rep 9(5):19081920. https://doi.org/10.1016/j.celrep.2014.11.013
21. Baser A, Skabkin M, Kleber S, Dang YL, Balta GSG, Kalamakis G, Gopferich M, Ibanez DC et al (2019) Onset of differentiation is post-transcriptionally controlled in adult neural stem cells. Nature 566(7742):100. https://doi.org/10.1038/s41586-019-0888-x

22. Noctor SC, Martinez-Cerdeno V, Kriegstein AR (2007) Contribution of intermediate progenitor cells to cortical histogenesis. Arch Neurol 64(5):639-642. https://doi.org/10.1001/archneur.64.5.639

23. Noctor SC, Martinez-Cerdeno V, Ivic L, Kriegstein AR (2004) Cortical neurons arise in symmetric and asymmetric division zones and migrate through specific phases. Nat Neurosci 7(2):136-144. https://doi.org/10.1038/nn1172

24. Kriegstein A, Alvarez-Buylla A (2009) The glial nature of embryonic and adult neural stem cells. Annu Rev Neurosci 32:149-184. https://doi.org/10.1146/annurev.neuro.051508.135600

25. Sen P, Shah PP, Nativio R, Berger SL (2016) Epigenetic mechanisms of longevity and aging. Cell 166(4):822-839. https://doi.org/ 10.1016/j.cell.2016.07.050

26. Bernstein BE, Mikkelsen TS, Xie X, Kamal M, Huebert DJ, Cuff J, Fry B, Meissner A et al (2006) A bivalent chromatin structure marks key developmental genes in embryonic stem cells. Cell 125(2):315-326. https://doi.org/10.1016/j.cell.2006.02.041

27. Bale TL (2015) Epigenetic and transgenerational reprogramming of brain development. Nat Rev Neurosci 16(6):332-344. https://doi. org/10.1038/nrn3818

Publisher's Note Springer Nature remains neutral with regard to jurisdictional claims in published maps and institutional affiliations. 